\title{
Medidas corporales y concentración sérica y folicular de lípidos y glucosa en vacas Brahman fértiles y subfértiles
}

\author{
Néstor Alonso Villa(1), Erik Felipe Pulgarín ${ }^{(1)}$, Paola Andrea Tabares $^{(1)}$, Eduardo Angarita ${ }^{(2)}$ y Alejandro Ceballos $^{(3)}$ \\ (1)Universidad de Caldas, Departamento de Sistemas de Producción, Calle 65\# 26-10, Manizales, Colombia. E-mail: navilla@ucaldas.edu.co \\ (2)Centro Genético La Cristalina, La Dorada, Colombia. E-mail: angarita5@hotmail.com (3)Atlantic Veterinary College, Department of Health \\ Management, 550 University Ave., Charlottetown, PE C1A 4P3, Canada. E-mail: aceballos@upei.ca
}

\begin{abstract}
Resumen - El objetivo de este estudio fue evaluar las diferencias en las medidas corporales en vacas Brahman fértiles y subfértiles, establecer las diferencias en la concentración de colesterol, lipoproteínas y glucosa en suero y líquido folicular, y desarrollar un modelo para predecir subfertilidad en vacas Brahman a partir de las medidas corporales. Se seleccionaron vacas Brahman registradas, agrupadas en fértiles (15) y subfértiles (15) según historial reproductivo. Se tomaron muestras de suero y líquido folicular (diámetro $>8 \mathrm{~mm}$ ) para determinar colesterol, lipoproteínas, triacilgliceroles y glucosa. Las vacas subfértiles mostraron un fenotipo masculino, con medidas corporales mayores, y concentraciones de colesterol y HDL séricos y foliculares más bajas que las vacas fértiles. El colesterol y el HDL se correlacionaron positivamente entre los compartimientos en ambos grupos. La subfertilidad es más probable en vacas pesadas con hombros amplios y mayor perímetro torácico. El tamaño corporal en vacas Brahman subfértiles se relacionó con su apariencia, con cambios en los metabolitos séricos y foliculares y con las concentraciones de colesterol y HDL.
\end{abstract}

Términos para indexación: Cebú, fertilidad, medición del cuerpo, metabolismo.

\section{Body measurements and serum and follicular concentrations of lipids and glucose in fertile and subfertile Brahman cows}

\begin{abstract}
The objective of this study was to evaluate differences in body size among Brahman cows, grouped according to their fertility, to establish differences in concentrations of cholesterol, lipoproteins, and glucose, in serum and follicular fluid, and to develop a model to predict subfertility in Brahman cows using body measurements as predictors. Registered Brahman cows were selected and grouped in fertile (15) and subfertile (15) animals, according to their calving interval. Samples of blood serum and liquid from follicles (diameter $>8 \mathrm{~mm}$ ) were collected in order to determine cholesterol, lipoproteins, triacylglycerols, and glucose. Subfertile cows had a masculine phenotype, their body measurements were larger than those in fertile cows, and they had lower cholesterol and HDL concentrations in serum and follicular fluid. Cholesterol and HDL between compartments were positively correlated in both groups. Subfertility is more probable in heavier cows with wider shoulders, and larger thoracic perimeter. Body size in subfertile Brahman cows was related to their appearance, and to changes in serum and follicular cholesterol and HDL concentrations.
\end{abstract}

Index terms: Zebu, fertility, body measurement, metabolism.

\section{Introducción}

El estado nutricional de los animales, reflejado en su condición corporal (CC), es determinante para mantener una adecuada función reproductiva. En ganado Brahman, se han documentado efectos críticos del tamaño corporal sobre las características reproductivas y productivas; así, los animales que poseen medidas bovinométricas moderadas tienen mejores índices de fertilidad (Vargas et al., 1999). Igualmente, se ha señalado que las vacas fértiles presentan diferencias en el fenotipo, en comparación con aquellas vacas con problemas de fertilidad (Bavera \& Peñafort, 2000).

El sobrepeso - entendido como un evidente incremento de la CC - está relacionado con cambios en el perfil lipídico y la subsecuente fertilidad en la hembra. Se ha observado una mayor lipólisis y acumulación de lípidos en el hígado, así como una correlación positiva entre la lipidosis hepática y el número de días para la primera ovulación postparto, en vacas con alta CC preparto (Rukkwamsuk et al., 1999). Investigaciones 
recientes han demostrado que el nivel de alimentación y su posterior efecto sobre la calidad de los ovocitos es dependiente de la CC del animal, en que un alto nivel de alimentación es benéfico para animales con una $\mathrm{CC}$ baja, mientras que este mismo régimen en animales sobrecondicionados induce un detrimento en la calidad del ovocito (Adamiak et al., 2005).

El régimen alimenticio en el preparto, los cambios metabólicos que acompañan el inicio de la lactancia, así como la CC preparto inducen cambios en la concentración sérica de diferentes metabolitos (Rukkwamsuk et al., 1999; Ciccioli et al., 2003). Estos cambios metabólicos en el suero son evidentes en la composición bioquímica del líquido folicular (Leroy et al., 2004a), y determinan a su vez la subsecuente calidad de los ovocitos y la fertilidad de la vaca (Leroy et al., 2004b). La glucosa es particularmente importante, ya que es el principal sustrato energético para el funcionamiento ovárico y requiere un aporte suficiente para mantener la actividad ovárica (Nishimoto et al., 2006). En la vaca se presentan fluctuaciones en la glicemia que podrían de una u otra forma afectar la concentración de glucosa en el líquido folicular (Leroy et al., 2004b), y que pueden estar relacionadas con cambios en el régimen alimenticio, o que correspondería a variaciones relacionadas con el estado fisiológico de la vaca (Rukkwamsuk et al., 1999; Ciccioli et al., 2003).

En Colombia, se han realizado estudios previos con respecto al balance de energía en ganado Brahman durante el pre y postparto (Osorio \& Escobar, 2002); sin embargo, no se ha descrito cuáles serían las eventuales diferencias en el metabolismo energético entre vacas fértiles y subfértiles, así como tampoco han sido descritas las diferencias en la composición bioquímica sérica y en el líquido folicular en los mismos grupos de vacas.

El objetivo de este estudio fue evaluar las eventuales diferencias en las medidas bovinométricas y en la concentración de colesterol, fracciones de colesterol y glucosa en el suero y líquido folicular en dos grupos de vacas Brahman, clasificados según su historial reproductivo. Asimismo, desarrollar un modelo para predecir la probabilidad de subfertilidad en vacas Brahman, a partir de sus medidas corporales.

\section{Materiales y Métodos}

Este estudio se realizó en el Magdalena Medio colombiano $\left(5^{\circ} 27^{\prime} \mathrm{N}, 74^{\circ} 40^{\prime} \mathrm{W}\right)$, con temperatura promedio de $28,5^{\circ} \mathrm{C}$, humedad relativa de $70 \%$ y precipitación anual de $1.200 \mathrm{~mm}$; la zona es clasificada como bosque tropical (Holdridge et al., 1971). El predio estaba ubicado a $178 \mathrm{~m}$ de altitud, en una explotación dedicada a la cría de ganado Brahman registrado. El estudio fue realizado inmediatamente despues de las pariciones de las vacas, lo que ocurrió entre los meses de Junio y Agosto, coincidente con la estación seca del año.

Los animales (30) fueron seleccionados según el historial reproductivo previo, divididos en dos grupos de 15 vacas. El primer grupo se clasificó como fértil, cuyo intervalo entre partos promedio fue $14,5 \pm 2,0$ meses, y edad media de 8 años. El segundo grupo, clasificado como subfértil, presentó intervalo entre partos promedio de $26,4 \pm 7,5$ meses y edad media de 7 años. La CC de las vacas, al momento de la evaluación, en la escala de 1 a 9 (Richards et al., 1986), fue 6 y 8 para los grupos fértil y subfértil, respectivamente. Las vacas se mantuvieron todo el tiempo en pastoreo directo en praderas de Angleton (Dichanthium aristatum), con contenido promedio de $10 \%$ de proteína cruda y energía metabolizable estimada en 1,8 Mcal kg-1 de materia seca, y además recibían un suplemento equivalente a $70 \mathrm{~g}$ por día de una mezcla de minerales con contenido promedio de $40 \%$ de cloruro de sodio, $8 \%$ de fósforo, $16 \%$ de calcio, $0,3 \%$ de magnesio, $4 \%$ de azufre, $0,01 \%$ de cobalto, $0,25 \%$ de cobre y $0,7 \%$ de zinc. El suministro de agua fue a voluntad.

Entre tres y cinco meses después del parto, en cada animal se midió: peso, amplitud de hombros, profundidad del pecho, perímetro torácico, distancia de la punta del pecho a la punta de la giba y amplitud de anca. Las medidas fueron tomadas mediante el uso de un bovinómetro y una cinta bovinométrica.

A las $7 \mathrm{~h}$ de la mañana y sin un ayuno previo, a cada vaca se le tomó una muestra de líquido folicular mediante punción ovárica, bajo seguimiento ecográfico con un ecógrafo ALOKA SD500 (Aloka Holding Europe AG, Zug, Suiza), equipado con un transductor convexo intravaginal de $5 \mathrm{MHz}$, y previa anestesia epidural. El líquido fue obtenido de los folículos dominantes en crecimiento y que presentaban diámetro superior a $8 \mathrm{~mm}$, y se 
envasó en tubos de reacción de 1,5 $\mathrm{mL}$, los cuales se congelaron posteriormente a $-20^{\circ} \mathrm{C}$ hasta su análisis. Paralelamente, se obtuvo una muestra de $10 \mathrm{~mL}$ de sangre sin anticoagulante, mediante venopunción coccígea con tubos al vacío (Vacutainer, Becton-Dickinson, Franklin Lakes, NJ, USA). Las muestras fueron centrifugadas inmediatamente, a $3.000 \mathrm{rpm}$ por $15 \mathrm{~min}$ para separar el suero; posteriormente, se envasó y conservó a $-20^{\circ} \mathrm{C}$ hasta su análisis en el Laboratorio de Patología Clínica y Reproducción, de la Universidad de Caldas, Colombia.

Tanto en el suero como en el líquido folicular se evaluó la concentración de glucosa (método GOD-PAP), colesterol (método enzimático CHOD-PAP), lipoproteínas de alta densidad (HDL, método fosfotungstato magnesio CHOD-PAP), lipoproteínas de baja densidad (LDL, ecuación de Friedewald), y triacilgliceroles (TAG, método enzimático GPO-PAP). Los análisis fueron realizados mediante espectrofotometría UV, con reactivos comerciales (BioSystems, Barcelona, España), y los resultados se expresaron en milimol por litro.

La comparación entre grupos, para las medias de las variables continuas, se realizó mediante un modelo lineal, con la evalución del efecto de la condición fértil o subfértil sobre las diferentes variables estudiadas, según el modelo: $y_{i}=\mu+$ $\alpha_{\text {condición(i) }}+\varepsilon_{i}$, en que: $y_{i}$ es el valor observado en las medidas corporales en los metabolitos sanguíneos o en líquido folicular, en la i-ésima vaca; $\mu$ es la media general; $\alpha_{(i)}$ es la condición fértil o subfértil de la i-ésima vaca; y $\varepsilon_{\mathrm{i}}$ es el error.

Posteriormente, con el objeto de predecir la respuesta de la vaca, en términos de su fertilidad potencial a partir de las medidas corporales, se utilizaron como regresores para la construcción de un modelo de regresión logística las medidas bovinométricas que presentaron diferencias entre ambos grupos de vacas, a $5 \%$ de probabilidad. Las medidas a incluir en el modelo se seleccionaron por pasos ("stepwise selection"), selección progresiva ("forward selection") y eliminación regresiva ("backward elimination"), y se fijó como criterio para la inclusión $\alpha \leq 0,10$ (Dohoo et al., 2003). El modelo final fue:

$\operatorname{logit}\left(\mathrm{p}_{\mathrm{i}}\right.$ fertilidad $)=\beta_{0}+\beta_{1} \mathrm{x}_{(\mathrm{pes})) \mathrm{ii}}+\beta_{2} \mathrm{x}_{(\mathrm{ah}) 2 \mathrm{i}}+\beta_{3} \mathrm{x}_{(\mathrm{pt}) 3 \mathrm{i}}$, en que: logit ( $p_{i}$ fertilidad) es el logaritmo natural de la probabilidad de ser subfértil; $\mathrm{x}_{1}$ es el peso $(\mathrm{kg})$; $\mathrm{x}_{2}$ es la amplitud de hombro $(\mathrm{cm}) ; \mathrm{x}_{3}$ es el perímetro torácico $(\mathrm{cm})$. Posteriormente, se evaluó la bondad de ajuste del modelo (Hosmer \& Lemeshow, 2000), y se determinaron la sensibilidad, la especificidad y el área bajo una curva de características funcionales (curva ROC: receiver operating characteristic curve), para el modelo final (Dohoo et al., 2003). El ajuste del modelo fue evaluado mediante la partición en dos grupos $(60 \%$ y $40 \%)$ de un número de valores generado aleatoriamente; el modelo fue reconstruido con el $60 \%$ de los datos, con la inclusión de los regresores descritos, y la predicción se evaluó con el $40 \%$ restante (Dohoo et al., 2003). Los análisis se hicieron mediante la utilización del paquete estadístico STATA 10.1 (StataCorp, College Station, TX, USA).

\section{Resultados y Discusión}

Si bien no se realizó un seguimiento de la CC de las vacas, la condición observada en las vacas subfértiles fue superior a la observada en las fértiles. Las diferentes medidas corporales estudiadas presentaron diferencias significativas entre ambos grupos de vacas (Cuadro 1). En promedio, las hembras subfértiles fueron $75 \mathrm{~kg}(13,7 \%)$ más pesadas que las vacas fértiles, y su aspecto se caracterizó por presentar excesivo depósito de grasa en el lomo, en el pecho, en la región de la cruz y la espalda, en comparación con medidas de las vacas fértiles. Esta condición les confirió a las hembras subfértiles un aspecto masculino, similar a las características descritas por Bavera y Peñafort (2000). En otros estudios, también se ha descrito que el tamaño corporal de la hembra tiene un marcado efecto sobre las características reproductivas. Las vacas con medidas bovinométricas moderadas

Cuadro 1. Promedio y error estándar (EE) de las medidas bovinométricas de dos grupos de vacas Brahman fértiles y subfértiles.

\begin{tabular}{lcrrr}
\hline Medida & Fértiles & Subfértiles & \multicolumn{1}{c}{ EE } & \multicolumn{1}{c}{$\mathrm{p}^{(1)}$} \\
\hline Peso (kg) & 548,1 & 623,2 & 16,20 & 0,003 \\
Amplitud de hombro (cm) & 40,3 & 48,0 & 1,08 & $<0,001$ \\
Punta de pecho - punta de giba (cm) & 80,7 & 89,1 & 1,90 & 0,004 \\
Profundidad (cm) & 70,9 & 74,4 & 0,98 & 0,017 \\
Perímetro torácico (cm) & 192,3 & 205,7 & 1,86 & $<0,001$ \\
Amplitud de anca (cm) & 52,4 & 57,5 & 0,90 & $<0,001$ \\
\hline
\end{tabular}

${ }^{(1)}$ Significancia estadística según el modelo de regresión lineal. 
presentan mejores índices de fertilidad (Vargas et al., 1999).

El sobrepeso y una mayor $\mathrm{CC}$ han sido asociados con alteraciones reproductivas, causando una apariencia externa masculina. La insulino-resistencia ocasiona cambios en la función ovárica en vacas obesas, alterando la aromatización periférica de andrógenos o disminuyendo la producción de la globulina transportadora de esteroides sexuales y aumentando la concentración circulante de esteroides libres. Igualmente, la leptina puede contribuir al desarrollo de insulino-resistencia (Poretsky et al., 1999), suprimiendo la esteroideogénesis inducida por la insulina en la granulosa y en las células tecales (Webb et al., 2004; Adamiak et al., 2005), o inhibir el sinergismo entre las gonadotropinas y la insulina (Spicer, 2001), asociándose con cambios en la función ovárica. Adicionalmente, el aumento en el consumo de energía induce cambios en la expresión de las proteínas ligadoras 2 y 4 de los factores de crecimiento similares a insulina, disminuyéndola en los folículos antrales pequeños (Webb et al., 2004). De otra parte, la incapacidad para alcanzar los requerimientos nutricionales en animales de mayor tamaño es otro factor que influye en las alteraciones del funcionamiento ovárico y de la fertilidad, mediante la reducción en la producción de insulina (Vargas et al., 1999; Butler et al., 2004; Webb et al., 2004). Esas alteraciones reproductivas, asociadas a los cambios en la insulina y leptina, han sido también descritas en mujeres y hembras obesas de otras especies (Poretsky et al., 1999; Spicer, 2001; Schneider, 2004). Estas alteraciones hormonales requieren la realización de otros estudios en vacas Brahman obesas con problemas de subfertilidad.

Una mayor circulación de estrógenos y andrógenos libres puede estar asociada con masculinización de la hembra, lo que causa una apariencia física masculina y resulta en las medidas observadas en las vacas subfértiles. Las interacciones entre la leptina, insulina, peso o $\mathrm{CC}$ y la función ovárica son complejas y contradictorias ocasionalmente (Schneider, 2004), así la hipo e hiperinsulinemia tienen efectos similares sobre la función ovárica mediados por diferentes mecanismos ocasionando las alteraciones descritas (Poretsky et al., 1999; Spicer 2001; Webb et al., 2004). También los animales de mayor tamaño tienen límites biológicos que deben considerarse, cuando se busca obtener la máxima fertilidad, no sólo para satisfacer sus requerimientos nutricionales, sino que hay interacciones entre el tamaño y la secreción hormonal que determinarán la función reproductiva potencial.

La concentración promedio de colesterol y HDL fue superior en las vacas fértiles comparado con las subfértiles (Cuadro 2). En el líquido folicular también se observaron diferencias significativas entre los grupos para los mismos metabolitos, siendo superior el promedio en las vacas fértiles (Cuadro 2). Las concentraciones en el suero fueron superiores a las observadas en el líquido folicular, excepto para la glucosa, cuya concentración folicular fue superior (Cuadro 2).

La concentración sérica de colesterol se correlacionó positivamente con la concentración folicular de HDL, tanto en las vacas fértiles $(r=0,72$; $\mathrm{p}<0,05)$ como subfértiles $(\mathrm{r}=0,62 ; \mathrm{p}<0,05)$, pero sólo en las fértiles las concentraciones sérica y folicular de HDL se relacionaron significativamente $(\mathrm{r}=0,79 ; \mathrm{p}<0,05)$. Para las demás correlaciones, entre los demás metabolitos, no hubo evidencia para aceptar su significancia $(\mathrm{p}>0,05)$.

La concentración promedio de colesterol estuvo dentro del valor referencial en el grupo de vacas fértiles, mientras que en el grupo subfértil fue inferior a la referencia (Osorio \& Escobar, 2002);

Cuadro 2. Promedio y error estándar (EE) de la concentración $\left(\mathrm{mmol} \mathrm{L}^{-1}\right)$ de metabolitos séricos, en líquido folicular de vacas Brahman fértiles y subfértiles.

\begin{tabular}{|c|c|c|c|c|c|c|c|c|}
\hline \multirow[t]{2}{*}{ Metabolito } & \multicolumn{4}{|c|}{ Suero } & \multicolumn{4}{|c|}{ Líquido folicular } \\
\hline & Fértiles & Subfértiles & $\mathrm{EE}$ & $p^{(1)}$ & Fértiles & Subfértiles & $\mathrm{EE}$ & $p$ \\
\hline Colesterol & 3,7 & 2,9 & 0,20 & 0,003 & 1,9 & 1,5 & 0,09 & 0,005 \\
\hline HDL & 3,0 & 2,4 & 0,16 & 0,011 & 1,5 & 1,0 & 0,09 & $<0,001$ \\
\hline LDL & 0,7 & 0,4 & 0,16 & 0,252 & 0,4 & 0,6 & 0,08 & 0,155 \\
\hline TAG & 0,4 & 0,4 & 0,02 & 0,389 & 0,1 & 0,1 & 0,01 & 0,775 \\
\hline Glucosa & 3,3 & 3,3 & 0,21 & 0,965 & 3,8 & 4,0 & 0,19 & 0,388 \\
\hline
\end{tabular}

${ }^{(1)}$ Significancia estadística según el modelo de regresión lineal. 
no obstante, los promedios en ambos grupos fueron superiores a lo descrito en otros estudios en vacas Brahman o en Cebú mestizas (Bastidas et al., 1990; Coppo et al., 2003). La concentración de HDL presentó diferencias entre los grupos, y sus valores promedio fueron similares a los descritos en otros estudios (Osorio y Escobar, 2002) o ligeramente superiores a otras observaciones en cebuínos (Coppo et al., 2003). Los de más metabolitos estudiados no presentaron diferencias entre los grupos, y sus valores fueron similares a los reportados en otros estudios (Bastidas et al., 1990; Osorio y Escobar, 2002; Coppo et al., 2003).

La concentración de colesterol tiene una relación directa con la alimentación de la vaca, debiendo ser superior en las vacas de mayor CC por un mayor consumo de energía (Bastidas et al., 1990; Bitman et al., 1990; Kida, 2003). Pese a ello, los resultados observados están en la dirección opuesta, ya que la colesterolemia y la concentración de HDL fueron inferiores en las vacas obesas (Cuadro 2). Lo anterior parece indicar que el colesterol sérico no sería dependiente únicamente del consumo de fibra efectiva $u$ otros precursores energéticos en la dieta, sino de nutrientes como la proteína cruda (Kida, 2003), y su consumo puede haber sido mayor en las vacas más delgadas. De otra parte, Kida (2003) señala que la CC refleja el balance de energía en el tiempo, mientras que la concentración sérica de colesterol o lipoproteínas refleja el balance de un momento más próximo a la evaluación sérica; así, las diferencias en la concentración de colesterol y HDL, en ambos grupos de vacas, podrían reflejar la alimentación recibida recientemente y no guardarían una relación directa con la $\mathrm{CC}$ de la vaca. Otro factor a considerar es la variación ultradiana en los lípidos séricos. Si bien las muestras en ambos grupos fueron tomadas a la misma hora, el colesterol hace picos cada 2,5 horas, con variaciones cercanas al $20 \%$ del promedio durante el día (Bitman et al., 1990).

La concentración folicular de colesterol y HDL presentó diferencias entre ambos grupos de vacas (Cuadro 2). En el grupo de vacas fértiles, la concentración folicular de colesterol fue similar a la observada en otros estudios, mientras que el promedio observado en el grupo de vacas subfértiles fue inferior al descrito (Leroy et al., 2004a, 2004b), lo que indica que la concentración folicular de colesterol estaría relacionada con el diámetro folicular (Leroy et al., 2004a). El colesterol folicular, en ambos grupos de vacas, fue un 52\% del colesterol sérico, lo que es coincidente con otros reportes (Leroy et al., 2004a, 2004b). El líquido folicular proporciona el ambiente propicio para el desarrollo del ovocito previo a la ovulación, y este compartimiento avascular está separado del estroma por la pared folicular (barrera hemato-folicular); no obstante, el líquido folicular es un exudado sérico, lo que indica que esta barrera es permeable a ciertos metabolitos durante el desarrollo del folículo; hay también una alta actividad metabólica in situ que sintetiza metabolitos localmente (Leroy et al., 2004a, 2004b). Como la barrera hemato-folicular es permeable al colesterol y al HDL, durante el desarrollo del folículo, e impermeable para otros lípidos, la concentración folicular está relacionada con la concentración sérica de estos metabolitos, lo que concuerda con los resultados observados en el presente estudio.

Un mayor intervalo entre partos, característica de las vacas subfértiles, puede estar relacionado con alteraciones de la síntesis hormonal en el ovario. Los esteroides ováricos dependen, para su formación, del aporte de colesterol obtenido de las lipoproteínas plasmáticas y de la síntesis local "de novo" con los precursores disponibles; además, se ha demostrado la expresión del RNAm, que codifica la presencia de los receptores específicos para las lipoproteínas que interiorizan los lípidos necesarios para la síntesis esteroide, en el ovario de la vaca (Argov \& Sklan, 2004). Así, una reducción en la concentración sérica de colesterol y HDL en el grupo de vacas subfértiles, que guarda relación directa con la concentración folicular, alteraría la disponibilidad de los precursores necesarios para la síntesis ovárica de esteroides, con el consecuente efecto sobre los índices reproductivos.

Las lipoproteínas plasmáticas LDL y VLDL son una fuente importante de colesterol para el líquido folicular y el posterior desarrollo del folículo (Argov \& Sklan, 2004). La concentración de lipoproteínas LDL y VLDL y triacilgliceroles, en el líquido folicular, resultan de procesos metabólicos locales, según el estado de desarrollo del folículo, no necesariamente relacionados con modificaciones de la dieta o el consumo de fuentes energéticas (Leroy et al., 2004a). 
Lo anterior puede estar relacionado con el hecho de que no se observaron diferencias en la concentración de triacilgliceroles entre los grupos de vacas en los dos compartimientos, lo que confirma que la concentración folicular de triacilgliceroles y otras lipoproteínas son procesos metabólicos localizados, cuyo estudio escapa a los objetivos de este trabajo.

La concentración sérica de glucosa fue inferior a lo observado en otros estudios (Landau et al., 2000; Leroy et al., 2004a); sin embargo, fue superior a lo descrito en vacas con alteraciones en la secreción de 17-beta-estradiol (Butler et al., 2004). El promedio para la glucosa sérica en ambos grupos de vacas fue inferior al promedio del líquido folicular (Cuadro 2), pese a ello, los valores foliculares coincidieron con reportes previos (Landau et al., 2000; Leroy et al., 2004b; Nishimoto et al., 2006), concentración que varía según el diámetro folicular (Leroy et al., 2004a) y la dieta que recibe la vaca (Landau et al., 2000).

La glucosa es una importante fuente energética para el funcionamiento ovárico, y está demostrado que la barrera hemato-folicular es permeable a ella, perola concentración sérica puede variar rápidamente debido a factores no dietéticos (Landau et al., 2000) o al estrés (Kida, 2003). Esto puede influenciar la concentración en el líquido folicular debido a la relación que hay entre ambos compartimientos (Leroy et al., 2004a, 2004b). Sin embargo, en este estudio, no se observó una correlación significativa entre la glicemia y la concentración de glucosa en el líquido folicular. Una concentración baja de glucosa en el líquido folicular, similar a la observada en el suero, puede resultar de un mayor número de células en la granulosa, por un desarrollo folicular menos avanzado, ya que las células de la granulosa demandan mayor cantidad de glucosa; además, la barrera hemato-folicular es más permeable a la glucosa en folículos de mayor diámetro (Leroy et al., 2004a), lo que puede comprobarse con estudios que relacionen la concentración en ambos compartimientos y que obtengan líquido de folículos con diámetros diferentes.

Con respecto al peso, si bien no se observaron diferencias, hubo una tendencia $(p=0,10)$ en las vacas con menor peso a una menor oportunidad relativa (OR: 0,94) de ser subfértiles (Cuadro 3). Las vacas con mayor amplitud de hombros y perímetro torácico tuvieron mayor probabilidad de ser subfértiles, OR: 2,21 $(\mathrm{p}=0,03)$ y OR: $1,90(\mathrm{p}=0,03)$, respectivamente (Cuadro 3). No se encontraron observaciones extremas que hubieran influenciado el modelo. La sensibilidad y especificidad del modelo, así como sus valores predictivos indicaron que los resultados predichos fueron correctos.

Las medidas corporales fueron diferentes entre ambos grupos de vacas (Cuadro 1), lo que aporta evidencia acerca de la asociación entre la conformación del animal y su desempeño reproductivo. Los regresores seleccionados fueron el peso, la amplitud de hombro y el perímetro torácico, para determinar la probabilidad de subfertilidad según las medidas corporales en vacas Brahman en pastoreo. Este modelo indicó que el riesgo para la ocurrencia de la condición de subfertilidad fue cercano al doble, en las vacas que presentaron mayor amplitud de hombro y perímetro torácico, asimismo el peso fue un factor asociado con el riesgo de ocurrencia del evento (Cuadro 3). Asociación esta biológicamente factible, en razón de la relación que existe entre las medidas corporales y la fertilidad (Bavera \& Peñafort, 2000; Westwood et al., 2002; Adamiak et al., 2005).

Cuadro 3. Modelo de regresión logística ${ }^{(1)}$ para fertilidad potencial en vacas Brahman.

\begin{tabular}{lrrrrr}
\hline Variable & Coeficiente & $\mathrm{p}^{(2)}$ & & $\mathrm{OR}^{(3)}$ & \multicolumn{2}{c}{ Intervalo de confianza de 95\% } \\
\cline { 5 - 6 } & & & & & Inferior \\
\hline Intercepto & $-123,41 \pm 50,48$ & 0,02 & & & 1,0 \\
Peso $(\mathrm{kg})$ & $-0,07 \pm 0,04$ & 0,10 & 0,94 & 0,9 & 4,5 \\
Amplitud de hombro $(\mathrm{cm})$ & $0,80 \pm 0,37$ & 0,03 & 2,21 & 1,1 & 3,3 \\
Perímetro torácico $(\mathrm{cm})$ & $0,63 \pm 0,29$ & 0,03 & 1,90 & 1,1 & \\
\hline
\end{tabular}

${ }^{(1)}$ Modelo basado en la selección progresiva o eliminación regresiva de las variables. ${ }^{(2)}$ Significancia estadística por la prueba de regresión logística. ${ }^{(3)}$ OR, oportunidad relativa. 


\section{Conclusión}

1. La apariencia masculina en vacas Brahman, determinada mediante las medidas corporales peso, amplitud de hombros y perímetro torácico, está relacionada con signos de subfertilidad establecidos mediante la alteración de los índices de fertilidad en la vaca.

2. El modelo presentado permite predecir la probabilidad de la ocurrencia del evento subfertilidad, a partir de las mediciones señaladas como regresores del evento.

3. Los cambios en el fenotipo del animal están asociados con cambios en el metabolismo.

\section{Referencias}

ADAMIAK, S.J.; MACKIE, K.; WATT, R.G.; WEBB, R.; SINCLAIR, K.D. Impact of nutrition on oocyte quality: cumulative effects of body composition and diet leading to hyperinsulinemia in cattle. Biology of Reproduction, v.73, p.918-926, 2005.

ARGOV, N.; SKLAN, D. Expression of mRNA of lipoprotein receptor related protein 8 , low density lipoprotein receptor, and very low density lipoprotein receptor in bovine ovarian cells during follicular development and corpus luteum formation and regression. Molecular Reproduction and Development, v.68, p.169-175, 2004.

BASTIDAS, P.; FORREST, D.W.; DEL VECCHIO, R.P.; RANDEL, R.D. Biological and immunological luteinizing hormone activity and blood metabolites in postpartum Brahman cows. Journal of Animal Science, v.68, p.2771-2778, 1990.

BAVERA, G.A.; PEÑAFORT, C. Evaluación exterior de los signos de fertilidad y subfertilidad de un rodeo. Río Cuarto: Universidad Nacional de Río Cuarto, 2000. 15p. Curso de Producción Bovina de Carne.

BITMAN, J.; WOOD, D.L.; LEFCOURT, A.M. Rhythms in cholesterol, cholesteryl esters, free fatty acids, and triglycerides in blood of lactating dairy cows. Journal of Dairy Science, v.73, p.948-955, 1990.

BUTLER, S.T.; PELTON, S.H.; BUTLER, W.R. Insulin increases 17ß-estradiol production by the dominant follicle of the first postpartum follicle wave in dairy cows. Reproduction, v.127, p.537-545, 2004.

CICCIOLI, N.H.; WETTEMANN, R.P.; SPICER, L.J.; LENTS, C.A.; WHITE, F.J.; KEISLER, D.H. Influence of body condition at calving and postpartum nutrition on endocrine function and reproductive performance of primiparous beef cows. Journal of Animal Science, v.81, p.3107-3120, 2003.

COPPO, N.B.; COPPO, J.A.; LAZARTE, M.A. Intervalos de confianza para colesterol ligado a lipoproteínas de alta y baja densidad en suero de bovinos, equinos, porcinos y caninos. Revista Veterinaria, v.14, p.3-10, 2003.

DOHOO, I.; MARTIN, W.; STRYHN, H. Veterinary epidemiologic research. Charlottetown: AVC, 2003. 706p.

HOLDRIDGE, L.R.; GRENKE, W.; HATHEWAY, W.H.; LIANG, T.; TOSI, J.A. Forest environments in tropical life zones: a pilot study. Oxford: Pergamon Press, 1971. 780p.
HOSMER, D.W.; LEMESHOW, S. Applied logistic regression. 2.ed. New York: John Wiley \& Sons, 2000. 392p.

KIDA, K. Relationships of metabolic profiles to milk production and feeding in dairy cows. Journal of Veterinary Medical Science, v.65, p.671-677, 2003.

LANDAU, S.; BRAW-TAL, R.; KAIM, M.; BOR, A.; BRUCKENTAL, I. Preovulatory follicular status and diet affect the insulin and glucose content of follicles in high-yielding dairy cows. Animal Reproduction Science, v.64, p.181-197, 2000.

LEROY, J.L.M.R.; VANHOLDER, T.; DELANGHE, J.R.; OPSOMER, G.; VAN SOOM, A.; BOLS, P.E.J.; DE KRUIF, A. Metabolite and ionic composition of follicular fluid from different-sized follicles and their relationship to serum concentrations in dairy cows. Animal Reproduction Science, v.80, p.201-211, 2004a.

LEROY, J.L.M.R.; VANHOLDER, T.; DELANGHE, J.R.; OPSOMER. G.;VANSOOM,A.;BOLS,P.E.J.;DEWULF,J.;DEKRUIF,A. Metabolic changes in follicular fluid of the dominant follicle in high-yielding dairy cows early post partum. Theriogenology, v.62, p.1131-1143, 2004b.

NISHIMOTO, H.; MATSUTANI, R.; YAMAMOTO, S.; TAKAHASHI, T.; HAYASHI, K.G.; MIYAMOTO, A.; HAMANO, S.; TETSUKA, $\mathrm{M}$. Gene expression of glucose transporter (GLUT) 1, 3 and 4 in bovine follicle and corpus luteum. Journal of Endocrinology, v.188, p.111-119, 2006.

OSORIO, J.M.; ESCOBAR, D.M. Determinación de los indicadores bioquímicos del balance energético durante el periparto de vacas Brahman en pastoreo. 2002. 25p. Tesis (Doctorado) - Universidad de Caldas, Manizales.

PORETSKY, L.; CATALDO, N.A.; ROSENWAKS, Z.; GIUDICE, L.C. The insulin-related ovarian regulatory system in health and disease. Endocrine Reviews, v.20, p.535-582, 1999.

RICHARDS, M.W.; SPITZER, J.C.; WARNER, M.B. Effect of varying levels of postpartum nutrition and body condition at calving on subsequent reproductive performance in beef cattle. Journal of Animal Science, v.62, p.300-306, 1986 .

RUKKWAMSUK, T.; WENSING, T.; KRUIP, T.A.M. Relationship between triacylglycerol concentration in the liver and first ovulation in postpartum dairy cows. Theriogenology, v.51, p.1133-1142, 1999.

SCHNEIDER, J.E. Energy balance and reproduction. Physiology and Behaviour, v.81, p.289-317, 2004.

SPICER, L.J. Leptin: a possible metabolic signal affecting reproduction. Domestic Animal Endocrinology, v.21, p.251-270, 2001.

VARGAS, C.A.; OLSON, T.A.; CHASE JUNIOR, C.C.; HAMMOND, A.C.; ELZO, M.A. Influence of frame size and body condition score on performance of Brahman cattle. Journal of Animal Science, v.77, p.3140-3149, 1999.

WEBB, R.; GARNSWORTHY, P.C.; GONG, J.G.; ARMSTRONG, D.G. Control of follicular growth: local interactions and nutritional influences. Journal of Animal Science, v.82, p.63-74, 2004.

WESTWOOD, C.T.; LEAN, I.J.; GARVIN, J.K. Factors influencing fertility of Holstein dairy cows: a multivariate description. Journal of Dairy Science, v.85, p.3225-3237, 2002.

Recibido el 10 de febrero de 2009 y aceptado el 3 de agosto de 2009 